\title{
Morphology of an autotomy fracture plane explains some of the variation in the latency to autotomize
}

\author{
Andrew Yoches \\ College of Agriculture and Life Science, University of Florida \\ Faculty mentor: Christine Miller, Department of Entomology and Nematology
}

\begin{abstract}
A gecko dropping its tail to escape predation beautifully illustrates the anti-predatory benefits associated with autotomy, i.e., the self-induced loss of a limb. The amount of time it takes an organism to drop its autotomizable limb can dramatically vary from one individual to the next, and the factors that are responsible for this variation remain unclear. Here, we investigated whether the size of the auototomy fracture plane, a morphological character, could explain variation in the latency to autotomize. We found that individuals with larger fracture planes took longer to autotomize when we statistically controlled for an individual's body size and sex. These results support previous assumptions about the relationship between fracture plane size and latency to autotomize. Namely, large individuals, with relatively small autotomy fracture planes, can drop their autotomizable limbs quickly, which likely improves their success in using this anti-predatory tactic.
\end{abstract}

Key Words: Anti-predation, Autotomy, Coreidae, Hemiptera

\section{Introduction}

Sacrificing an appendage to escape predation is frequently associated with geckos that drop their tails. However, this anti-predatory trait, termed autotomy, is also observed in several other taxa, such as insects, crabs, and mollusks (Maginnis, 2006; Fleming et al., 2007). In addition to autotomy's anti-predatory benefit, autotomy can also be used to escape non-predatory entrapment (Maginnis 2008) and to reduce the cost of injury (Emberts et al, 2017). In all of these cases, dropping a limb comes with an immediate fitness benefit, but limb loss also comes with a cost. These costs may include reduced locomotive ability, decreased energy stores, and fewer mating opportunities (Webb et al., 2006; Fleming et al., 2007). The cost of losing a limb is also a factor in an individual's decision to autotomize. While autotomy has a behavioral component, there is a morphological component as well. Most perceptibly, autotomy occurs at a specific breakage plane (Luscher, 1948). Both economic and optimality models of escape assume that initiating escape more quickly increases an individual's probability of successfully evading a 
predator (Ydenberg \& Dill, 1986; Cooper \& Frederick, 2007; Black et al., 2019; Emberts et al., 2019). Therefore, factors that affect the amount of time it takes an individual to autotomize could determine the difference between life and death.

Previous research on autotomy has focused on either the autotomy fracture plane or the latency to autotomize (Fox et al., 1998; Bose, 2013), but both components have rarely been investigated simultaneously. From a biomechanics perspective, larger fracture planes require more force to break for a given auototmizable appendage (Fox et al., 1994; Fox et al.,1998, Marrs et al., 2000, Gleason et al., 2014, but see Prestholdt 2018 for force difference across autotomizable appendages, e.g., legs vs. claws). It is unclear, however, whether the size of the fracture plane affects the latency to autotomize. Individuals with larger fracture planes may, for example, have compensatory muscles that allow them to autotomize their limbs just as quickly. The lack of studies investigating the relationship between morphology and behavior is problematic because previous work has assumed that there is a positive relationship between the size of an autotomy fracture plane and the associated behavior - latency to autotomize. The aim of this study is to understand the degree to which autotomy fracture planes affect the latency to autotomize.

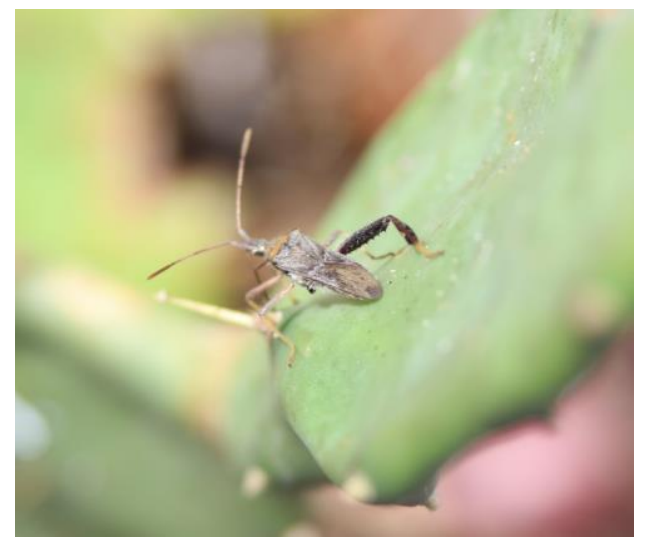

Figure 1: A male Narnia femorata missing his hind left leg. The leg was removed at the individual's autotomy fracture plane, which suggests that the limb was lost through autotomy (Miller).

We used the leaf-footed cactus bug Narnia femorata to investigate this aim. Like other coreids, $N$. femorata can drop their limbs to escape compromising situations (Figure 1). Moreover, autotomy in this species is frequently induced within 60 seconds, suggesting that autotomy is used as an anti-predatory trait (Emberts et al., 2016). Although autotomizing limbs 
to escape predation has yet to be experimentally demonstrated in this species, N. femorata has been observed successfully, and unsuccessfully, using autotomy to escape predation from a variety of predators including lizards and active foraging spiders. When inducing autotomy, limbs are always dropped at the same location, the trochanter-femur joint, which we will refer to as the autotomy fracture plane. In the wild, approximately $10 \%$ of $N$. femorata are missing at least one limb at this location (Emberts et al., 2016). The frequency and speed at which limbs are dropped, as well as their morphological variation (i.e., sexual dimorphism in hind limbs and variation in body size), make $N$. femorata an ideal system to investigate whether the size of the autotomy fracture plane affects the latency to autotomize. Since research in other species has shown that larger fracture planes take more force to break for a given autotomizable appendage, we hypothesized that there would be a positive relationship between the size of the autotomy fracture plane and the latency to autotomize.

\section{Methods}

\section{Study Design}

For our experiment, we used second generation individuals taken from a lab population of $N$. femorata. The lab population was founded in June of 2017 with wild caught adults collected from Live Oak, Florida $\left(30.26^{\circ} \mathrm{N},-83.18^{\circ} \mathrm{W}\right)$. All second generation individuals were reared on an intermediate quality diet (i.e. Opuntia mesacantha ssp. lata cladodes and unripe fruit) with their clutch siblings until their $4^{\text {th }}$ instar, to simulate a natural developmental environment. Upon reaching their fourth instar, we isolated individuals and randomly placed them into one of three rearing environments: cactus pad with no fruit (i.e., a low quality diet; $n=33$ ), cactus pad with unripe fruit (i.e., an intermediate quality diet; $\mathrm{n}=36$ ), and cactus pad with ripe fruit (i.e., a high quality diet; $n=46$ ). These three naturally occuring environments allowed us to generate morphological variation in $N$. femorata. Individuals were monitored every other day to assess development. Once individuals completed their terminal molt they were given at least 24 hours to sclerotize before being used in the experiment. Adults over 8 days old were not used. All individuals were reared in a greenhouse with a set temperature of 21-32 degrees Celsius and a 14:10 h L:D photoperiod.

We induced autotomy by gripping an individual's hind right femur with reverse action forceps to simulate a predation event. Reverse action forceps were used for a controlled grip strength 
during each trial. Trials were conducted by two observers, one timer and one experimenter. The timer used a handheld stopwatch to capture the latency to autotomize. Experimental trials were constrained to 15 minutes. Individuals who did not autotomize within the 15 min timeframe (in our case 2 out of the 115 individuals) were recorded as autotomizing at the 15 min mark, following previously established protocol (Bateman \& Fleming 2008; Pears et al., 2018). Each trial was conducted at a temperature of 27 degrees Celsius. After experiment trials were conducted, each individual was photographed using a Canon EOS 50D digital camera attached to a Leica M 165C dissecting microscope. Maximum pronotal width (PW; i.e., a well estiablished body size proxy for this species; Procter et al. 2012) and the diameter of the trochanter femur joint (i.e., fracture plane size) were measured using ImageJ v1.46. Pronotal width was measured from an aerial photograph and trochanter femur joint was measured from a horizontally-angled photograph to capture diameter.

\section{Statistical Analyses}

To investigate the effect of the autotomy fracture plane on the latency to autotomize we employed the use of generalized linear models (GLMs). Because other studies have investigated the latency to autotomize through a bivariate approach, we ran multiple GLMs with one explanatory variable at a time including size of autotomy fracture plane, body size, sex, and diet. These variables were selected because they have previously been shown, in other species, to influence the latency to autotomize (Daniels, 1984; Fox et al., 1998; Marrs et al., 2000). We specifically took this bivariate approach first, so that we could compare our results with previous studies. However, we also used a more comprehensive approach and included all explanatory variables (as well as all pairwise interactions) in a single model. We then simplified that model by using stepwise reduction with the R function step (R Core Team 2017), which evaluates models based on Akaike information criterion (Hastie \& Pregibon 1992; Venables \& Ripley 2002). For all of our analyses we used a $\log _{\mathrm{e}}$ transformation on the latency to autotomize (i.e., our response variable). Analyses were conducted in R version 3.4.2 (R Core Team 2017).

\section{Results}

When we took the bivariate approach, we found that individuals with larger fracture planes autotomize their limbs more quickly (i.e., a negative correlation; Figure $2 \mathrm{a} ; \mathrm{F}_{1,112}=13.801, \mathrm{R}^{2}=$ 
$0.102, p<0.001$ ). However, larger individuals also have larger autotomy fracture planes (Figure 2b; males: $\mathrm{F}_{1,50}=369.18, \mathrm{R}^{2}=0.878, p<0.001$; females: $\mathrm{F}_{1,60}=240.72, \mathrm{R}^{2}=0.797, p<0.001$ ), and the observed correlation could be reflecting that larger individuals autotomize more quickly $\left(F_{1,112}=33.70, R^{2}=0.224, p<0.001\right)$. The strength of the more comprehensive approach is the potential to tease apart such effects. This multivariate approach revealed (Table 1), when controlling for other variables such as body size and sex, that individuals with larger fracture planes autotomized their limbs more slowly (i.e., a positive correlation; Figure $2 c ; F_{1,104}=4.513$, $p=0.036$, Table 2).

Our multivariate model also revealed that $\operatorname{sex}\left(\mathrm{F}_{1,104}=4.210, p=0.042\right)$ and $\operatorname{diet}\left(\mathrm{F}_{1,104}=9.574\right.$, $p<0.001)$ explain additional variation in the latency to autotomize. Specifically, when controlling for the other variables in the model, males autotomize their limbs more quickly. This sex difference, however, was not observed in our bivariate analysis $\left(\mathrm{F}_{1,113}=0.001, p=0.972\right)$. The effect of diet, on the other hand, was observed in both the multivariate $\left(\mathrm{F}_{1,104}=9.574, p<\right.$ $0.001)$ and the bivariate analyses $\left(\mathrm{F}_{2,112}=26.353, p<0.001\right)$; those reared on a low quality diets took longer to autotomize. Furthermore, there were significant interactions between diet and both of our morphological traits (Diet x Fracture Plane Size: $\mathrm{F}_{2,104}=3.974, p=0.022$; Diet x Body Size: $\mathrm{F}_{2,104}=3.166, p=0.046$ ), which can make it challenging to interpret these main effects (i.e., the effects of diet, body size, and fracture plane size). Moreover, including diet treatment into our model resulted in multicollinearity. Therefore, we subsetted the data by diet and ran multivariate, a posteriori, analyses using the remaining explanatory variables (body size, fracture plane size, and sex) on each diet seperately.

The relationships between the response and explanatory variables were the same across all three diets. Larger individuals autotomize more quickly, individuals with larger fracture planes autotomize more slowly, and males autotomize more quickly (Table 3). However, sex did not significantly explain additional variation in the latency to autotomize in any of these models (Table 3; low quality diet: $\mathrm{F}_{1,29}=1.496, p=0.231$; intermediate quality diet: $\mathrm{F}_{1,32}=2.266, p=$ 0.142 ; high quality diet: $\mathrm{F}_{1,41}=0.490, p=0.488$ ). Furthermore, the morphological relationships with the latency to autotomize were only significant for the poor diet treatment (Table 3; Body Size: $\mathrm{F}_{1,29}=8.412, p=0.007$; Fracture Plane Size: $\mathrm{F}_{1,29}=7.852, p=0.009$ ). 

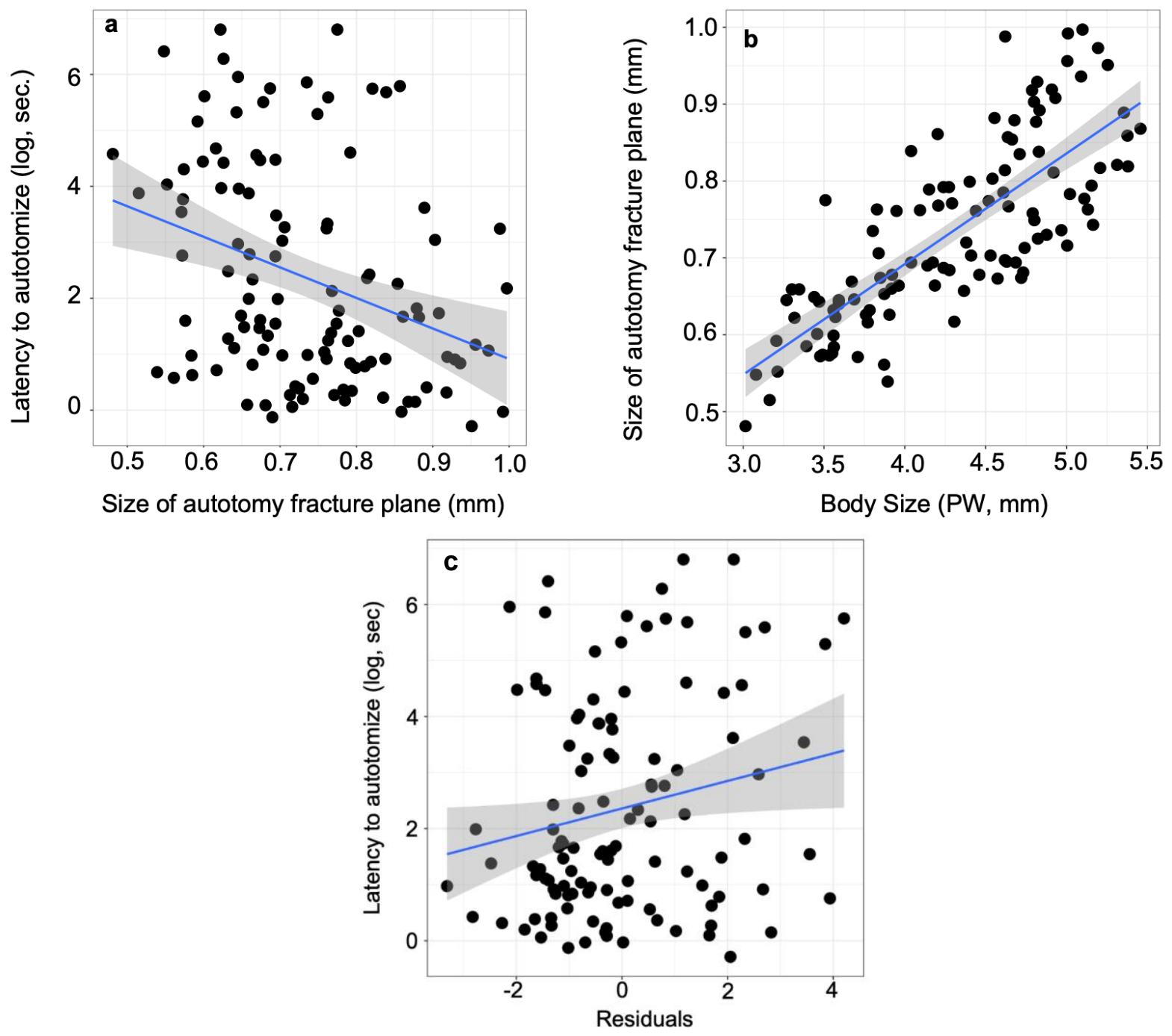

Figure 2a. The negative relationship between latency to autotomize and size of autotomy fracture plane. Figure $\mathbf{2 b}$. The positive relationship between size of autotomy fracture plane and body size. PW refers to pronotal width, a common body size proxy in insects. Figure 2c. A visual representation of the positive relationship between the size of autotomy fracture planes (when statistically controlling for other variables) and the latency to autotomize. Full model residuals without the fracture plane variable are displayed.

Table 1. Final step of our stepwise model reduction analysis.

\begin{tabular}{lc}
\hline Variables & AIC \\
\hline None & $\mathbf{4 3 3 . 1 1}$ \\
Sex & 435.63 \\
Body Size x Diet & 435.85 \\
Fracture Plane Size x Diet & 437.50 \\
\hline
\end{tabular}


Table 2. Reduced multivariate model: $\log _{\mathrm{e}}$ (Latency to Autotomize $) \sim$ Sex + Body Size + Fracture Plane Size + Diet + Body Size x Diet + Fracture Plane Size x Diet.

\begin{tabular}{lcccc}
\hline & $\begin{array}{c}\text { Esitmated } \\
\text { Coefficients }\end{array}$ & $\begin{array}{c}\text { Standard } \\
\text { Error }\end{array}$ & $\mathrm{t}$ value & $p$ \\
\hline Intercept & 7.0751 & 2.6703 & 2.650 & 0.009 \\
Sex & -0.9779 & 0.4766 & -2.052 & 0.043 \\
Body Size & -3.7029 & 1.0781 & -3.435 & $<0.001$ \\
Fracture Plane Size & 17.0608 & 5.1851 & 3.290 & 0.001 \\
Intermediate Quality Diet & -1.8270 & 3.3601 & -0.544 & 0.588 \\
High Quality Diet & -2.7492 & 3.4704 & -0.792 & 0.430 \\
Intermediate Quality Diet x & 2.9116 & 1.1584 & 2.513 & 0.013 \\
Body Size & & & & \\
High Quality Diet & 2.1083 & 1.1684 & 1.804 & 0.074 \\
x Body Size & & & & \\
Intermediate Quality Diet x & -16.2222 & 5.7545 & -2.819 & 0.006 \\
Fracture Plane Size & & & & \\
High Quality Diet & -10.7308 & 5.4243 & -1.978 & 0.051 \\
x Fracture Plane Size & & & & \\
\hline
\end{tabular}

Table 3: Results of the relationships between specfic traits and latency to autotomize for 3 separate models (HQD)

\begin{tabular}{lccc}
\hline & Estimated Coefficients & $F$ & $p$ \\
\hline HQD - Body Size & -1.2532 & 2.3968 & 0.1293 \\
HQD - Sex & -0.5356 & 0.4897 & 0.4880 \\
HQD - Fracture Plane Size & 4.0127 & 4.7472 & 0.3924 \\
IQD - Body Size & -1.3942 & 1.3195 & 0.2592 \\
IQD - Sex & -1.691 & 1.2662 & 0.1420 \\
IQD - Fracture Plane Size & 4.661 & 0.4193 & 0.5219 \\
LQD - Body Size & -3.5860 & 8.4125 & 0.0070 \\
LQD - Sex & -0.8668 & 1.4963 & 0.2311 \\
LQD - Fracture Plane Size & 16.5238 & 7.8521 & 0.0100 \\
\hline
\end{tabular}

High Quality Diet, IQD: Intermediate Quality Diet, and LQD: Low Quality Diet). Negative coefficients indicate negative correlation between pronotal width or fracture plane size and latency to autotomize. For sex, a negative value indicates males autotmized quicker, when controlling for body size and fracture plane size.

\section{Discussion}

It has previously been assumed that there is a positive correlation between autotomy fracture planes size and the latency to autotomize. In this study, we found support for this correlation (Figure 2c). However, this relationship only held when we statistically controlled for other covariates, such as body size. In fact, when we investigated the correlation between autotomy fracture plane size and the latency to autotomize in a bivariate analysis we found a negative correlation (Figure 2a). This result highlights the limitations of interpreting ecological function from biomechanical studies alone. Namely, understanding the natural history of the organism is 
of utmost importance. In this case, having a larger fracture plane may require more force to break, but individuals with larger fracture planes are larger individuals. These larger individuals, in general, must generate more force to autotomize compared to smaller individuals to explain how larger individuals are able to autotomize more quickly.

Morphology has previously been shown to influence the latency to autotomize. Namely, larger damselfly, starfish, and cricket individuals tend to autotomize more slowly or require more force to autotomize (Marrs et al., 2000; Bateman and Fleming, 2008). In fact, increased body size has been hypothesized to result in autotomy being secondarily lost (Arnold, 1984). However, in our study, we found that larger individuals autotomized more quickly. Thus we found the opposite pattern of what has been previously observed. The observed differences may be due to confounding variables such as diet, energy constraints, and muscle capacity. Such inconsistency calls for more research to be conducted on the relationship between morphology and behavior in autotomy. Specifically, the anatomy of autotomy in N. femorata has not been widely studied and a better understanding of the physiology associated with this trait could help explain our observed patterns.

Narnia femorata found in central Florida, such as our study population, naturally feed off of Optunia humifusa cactus pads and fruit. In nature, N. femorata face competition for this cactus fruit with large rodents and other mammals, leaving $N$. femorata with a discontinuous supply (Miller \& De Lestang, 2009). Our study tried to simulate this nutitional pattern in order to create naturally occurring morphological differences. It was apparent these specific diets have an impact on latency to autotomize, even when controlling for morphology. The subsequent effects of the diet treatments (besides morphology) were not the objective of the study, but are a beneficial additions to the research. The influence of diet on autotomy has also been noted in the gecko Phyllodactylus marmoratus. The study found that starved geckos took longer to autotomize than well fed geckos (Daniels, 1984). Similarly, we found that those reared in the poorest nutritional environment took the longest to autotomize even when controlling for morphological variation.

We also found, when controlling for morphology, that males autotomize their hind legs more quickly. Sex differences in the latency to autotomize have previously been observed in other species (Fox et al., 1998; Wasson \& Lyon, 2005). In these cases, the differences in autotomy were attributed to difference in predation pressure or differences in the cost of losing the 
autotomized limb. Other research has shown that male N. femorata use their hind legs in intrasexual competitions (Proctor et al., 2012; Nolen et al., 2017), and the loss of these hind legs decreases a male's probability of winning a fight (Emberts et al., 2018). Additionally, males that lose these sexually selected weapons have decreased mating success. These negative effects may make males less likely to drop their hind legs. Comparatively, female hind legs are thought to only serve as a locomotive function. Despite this sex specific costs of losing a sexually selected weapon, we found (when controlling for morphology) that males autotomize their hind legs more quickly. However, when we were not controlling for morphology there was no observable sex difference in the latency to autotomize. A majority of males (52\%) and females (54\%) autotomized their hind leg after being entrapped for 6 seconds (female median: 4.94s, male median: $5.53 \mathrm{~s})$. We interpret this to mean that there is strong selection, likely from predation, for both sexes to autotomize by that time (i.e., within 6s). However, since there are morphological differences between males and females, and since morphology influences the latency to autotomize (see above), males must behave differently (i.e., more quickly) for the functional outcome of the trait to be expressed within 6s.

In summary, fracture plane and body size, two morphological traits, could explain some of the variation in the latency to autotomize. Diet also influence the speed at which an organism autotomized their appendage. This study is a first step in explaining why we observe so much variation in the latency to autotomize. One unifying framework that could potentially explain these patterns is that the role of an organisms muscles in initiating autotomy. Most notably, those reared on the highest quality quality diet have larger hind leg muscles and autotomized the quickest. Similarly, males, which have larger hind leg muscles when compared to females, autotomized the quickest. Thus, variation in the latency to autotomize could be strongly linked with energy availability and muscle mass. However, other factors responsible for this pattern can not be excluded and future work is merited.

\section{Acknowledgements}

We would like to thank the members of the Miller, St. Mary, and Keiser labs for the insights they provided. We would also like to thank the anonymous reviewers of this manuscript for their remarks. This research was funded, in part, by the National Science Foundation grant IOS-1553100 to CWM. 


\section{References}

Arnold, E. N. (1984). Evolutionary aspects of tail shedding in lizards and their relatives. Journal of Natural History. 18, 127-169.

Bateman, P. W. \& Fleming, P. A. (2008). An intra- and interspecific study of body size and autotomy as a defense in Orthoptera. Journal of Orthoptera Research. 17, 315-320.

Black, K. L., Fudge, D., Jarvis, W. M., \& Robinson, B. W. (2019). Functional plasticity in lamellar autotomy by larval damselflies in response to predatory larval dragonfly cues. Evolutionary Ecology. 1-16.

Bose, A. P. H. \& Robinson, B. W. (2013). Invertebrate predation predicts variation in an autotomy-related trait in larval damselfly. Evolutionary Ecology. 27, 27-38.

Cooper, W. E. \& Frederick, W. G. (2007). Optimal flight initiation distance. Journal of Theoretical Biology. 244, 59-67.

Daniels, C. (1984). The Importance of Caudal Lipid in the Gecko Phyllodactylus marmoratus. Herpetologica. 40, 337-344.

Emberts, Z., Escalante, I., \& Bateman, P. W. (2019) The ecology and evolution of autotomy. Biological Reviews.

Emberts, Z., St. Mary, C. M., \& Miller, C. W. (2016). Coreidae (Insecta: Hemiptera) limb loss and autotomy. Annals of the Entomological Society of America. 109, 678-683.

Emberts, Z., St. Mary, C. M., Miller, C. W., \& Kiehl, D. (2017). Cut your Losses: self-amputation of inured limbs increases survival. Behavioral Ecology. 28, 1047-1054.

Emberts, Z., St. Mary, C. M., Herrington, T. J., \& Miller, C. W. (2018). Males missing their sexually selected weapon have decreased fighting ability and mating success in a competitive environment. Behavioral Ecology and Sociobiology. 72, 81.

Fleming, P. A., Muller, D., \& Bateman, P. W. (2007). Leave it all behind: a taxonomic perspective of autotomy in invertebrates. Biological Reviews. 82, 481-510.

Fox, S. F., Conder, J. M., \& Smith, A. E. (1998). Sexual dimorphism in the ease of tail autotomy: Uta stansburiana with and without previous tail loss. Copeia. 1998, 376-382.

Fox, S. F., Perea-Fox, S., \& Franco, R. C. (1994). Development of the tail autotomy adaptation in lizards under disparate levels of predation at high and low elevations in Mexico. The Southwestern Naturalist. 39, 311-322.

Gleason, J., Fudge, D., \& Robinson, B. (2014). Eco-mechanics of lamellar autotomy in larval damselflies. Journal of Experimental Biology. 217, 185-191.

Hastie, T. J. \& Pregibon, D. (1992). Generalized linear models. Statistical Models in S eds J. M. Chambers and T. J. Hastie, Wadsworth \& Brooks/Cole. 
Luscher, M. (1948). The regeneration of legs in Rhodnius prolixus (Hempiptera). Journal of Experimental Biology. 25, 334-343.

Maginnis, T. L. (2006). The costs of autotomy and regeneration in animals: a review and framework for future research. Behavioral Ecology. 17, 857-872.

Maginnis, T. L. (2008). Autotomy in a Stick Insect. Florida Entomologist. 91, 126-127.

Marrs, J., Wilkie, I. C., Skold, M., Maclaren, W. M., \& McKenzie, J. D. (2000). Size-related aspects of arm damage, tissue mechanisms, and autotomy in the starfish Asterias rubens. Marine Biology. $137,59-70$

Miller, C. W. \& De Lestang, F. N. (2009). Effects of Diet on Development and Surviviorship of Narnia femorata Nymphs. Florida Entomological Society. 92, 511-512.

Nolen, Z., Allen, P., \& Miller, C. W. (2017). Seasonal resource value and male size influence male aggressive interactions in the leaf footed cactus bug, Narnia femorata. Behavioral Processes. 138, $1-6$.

Pears, J. B., Emberts, Z., \& Bateman, P. W. (2018). The scent of danger: the impact of predator chemical cues on emergence from refuge and willingness to autotomize limbs in the house cricket (Acheta domesticus). Journal of insect behavior. 31(4), 416-426.

Prestholdt, T. E., Rager, S. M., Williams, J. N., \& Weilhoefer, C. L. (2018). The force needed for leg autotomy in the crab Hemigrapsus nudus. Marine and Freshwater Behaviour and Physiology. 51, $175-181$.

Procter, D. S., Moore, A. J., \& Miller, C. W. (2012). The form of sexual selection arising from male-male competition depends on the presence of females in the social environment. Journal of Evolutionary Biology. 25, 803-812.

Venables, W. N. \& Ripley, B. D. (2002). Modern Applied Statistics with S. New York: Springer. 4.

Wasson, K. \& Lyon, B. E. (2005). Flight or fight: flexible antipredatory strategies in porcelain crabs. Behavioral Ecology. 16, 1037-1041.

Webb, J. K. (2006). Effects of tail autotomy on survival, growth and territory occupation in free-ranging juvenile geckos (Oedura lesueurii). Austral Ecology. 31, 432-440

Ydenberg, R. C. \& Dill, L. M. (1986). The economics of fleeting from predators. Advances in the Study of Behavior. 16, 229-249. 